\title{
Modeling of convection, temperature distribution and dendritic growth in glass-fluxed nickel melts
}

\author{
Jianrong Gao ${ }^{\mathrm{a}, *}$, Andrew Kao ${ }^{\mathrm{b}}$, Valdis Bojarevics ${ }^{\mathrm{b}}$, Koulis Pericleous ${ }^{\mathrm{b}}$, Peter K. Galenko ${ }^{\mathrm{c}}$, \\ Dmitri V. Alexandrov ${ }^{\mathrm{d}}$ \\ ${ }^{a}$ Key Laboratory of Electromagnetic Processing of Materials (Ministry of Education), Northeastern University, Shenyang 110819, China \\ b Center of Numerical Modeling and Process Analysis, University of Greenwich, London SE10 9LS, United Kingdom \\ ${ }^{c}$ Department of Physics and Astronomy, Friedrich Schiller University of Jena, Jena 07743, Germany \\ ${ }^{\mathrm{d}}$ Department of Mathematical Physics, Ural Federal University, Ekaterinburng 620000, Russian Federation
}

\section{A R T I C L E I N F O}

Communicated by: Andrea Zappettini

\section{Keywords:}

A1. Dendrites

A1. Convection

A1. Impurities

A1. Solidification

A2. Growth from melts

B1. Metals

\begin{abstract}
A B S T R A C T
Melt flow is often quoted as the reason for a discrepancy between experiment and theory on dendritic growth kinetics at low undercoolings. But this flow effect is not justified for glass-fluxed melts where the flow field is weaker. In the present work, we modeled the thermal history, flow pattern and dendritic structure of a glass-fluxed nickel sample by magnetohydrodynamics calculations. First, the temperature distribution and flow structure in the molten and undercooled melt were simulated by reproducing the observed thermal history of the sample prior to solidification. Then the dendritic structure and surface temperature of the recalescing sample were simulated. These simulations revealed a large thermal gradient crossing the sample, which led to an underestimation of the real undercooling for dendritic growth in the bulk volume of the sample. By accounting for this underestimation, we recalculated the dendritic tip velocities in the glass-fluxed nickel melt using a theory of three-dimensional dendritic growth with convection and concluded an improved agreement between experiment and theory.
\end{abstract}

\section{Introduction}

Dendritic growth in undercooled melts has been the subject of intensive studies for fundamental and technical interest [1-3]. Advances in techniques and instrumentation for in-situ observations of rapid solidification [4] provided novel opportunities to test theories of two-dimensional or three-dimensional dendritic growth relying on purely diffusive or convective transport of heat and mass [5-7]. Studies showed that there exists a discrepancy between experiment and theory regarding dendritic growth kinetics of pure substances at low undercoolings. This discrepancy was often correlated to natural or forced convection in undercooled melts [8-10]. While continuous efforts were made for a precise knowledge of forced convection in electromagnetically levitated melts [11-13], little work has been done on the forced convection present in glass-fluxed melts. In principle, the use of induction heating in the glass-fluxing experiments should produce similar flow patterns in molten samples. But, the glass-fluxing experiments do allow for a reduction or even a turning-off of the heating power during cooling of the molten samples, since there is no need to levitate the sample mass against gravity. As a result, one may expect a reduced flow velocity and perhaps a reduced dendritic tip velocity in the glass-fluxed samples at similar undercoolings. Nevertheless, measured tip velocities in a glass-fluxed sample of pure nickel were found to be even higher than those in the electromagnetically levitated samples [10]. To interpret such higher tip velocities, a flow velocity incident to the growth direction of primary tips as high as $3 \mathrm{~m} / \mathrm{s}$ would have to be operative in the glass-fluxed sample. Such a flow velocity is 10 times higher than a generally accepted value of $\sim 0.3 \mathrm{~m} / \mathrm{s}$ for terrestrial electromagnetically levitated samples [11-13] and thus unlikely to be realistic. In the present work, we performed numerical simulations of flow patterns, temperature distribution and dendritic structure of a glass-fluxed sample of pure nickel. The results of the simulations allowed us to explore the reasons for the higher tip velocities observed at low undercoolings [10].

\section{Modeling of heat transfer and flow structure}

The temperature distribution and flow structure of inductively melted samples depends on material properties and experimental set-up. For this reason, we give a brief overview of the experimental setup used in the recent glass-fluxing experiment on a sample of pure nickel

\footnotetext{
* Corresponding author.

Email address: jgao@mail.neu.edu.cn (J. Gao)
} 
[10]. The nickel sample had a mass of $1 \mathrm{~g}$ and a nearly-spherical geometry with a diameter of $6 \mathrm{~mm}$. The sample was seated on a pan-like alumina-made holder containing soda lime glass, and was positioned in the coil of a radio frequency induction melting furnace. As shown in Fig. 1, the coil consists of top and lower parts, both of which have a cylindrical geometry. The top part of the coil has two windings, and the lower part has five opposite windings. Individual windings have an inner diameter of $33 \mathrm{~mm}$ and an outer diameter of $41 \mathrm{~mm}$. The shortest distance between the top part and the lower part is about $8.8 \mathrm{~mm}$, whereas the total height of the coil is $48.2 \mathrm{~mm}$. The sample was inductively heated, melted, and overheated under the protection of an argon atmosphere of high purity. The radio frequency generator of the induction melting furnace was switched to a high power for rapid heating of the sample. Then, the power was reduced to about $14 \%$ of its initial value. The sample was cooled by radiating to the water-cooled coil and to the chamber of the furnace. Solidification set in spontaneously when the sample was undercooled below its melting temperature. Rapid release of the latent heat produced a detectable recalescence event. The recalescence event usually commenced on the lower surface of the sample and spread towards the opposite side. The sample was not fully solidified until an isothermal process followed

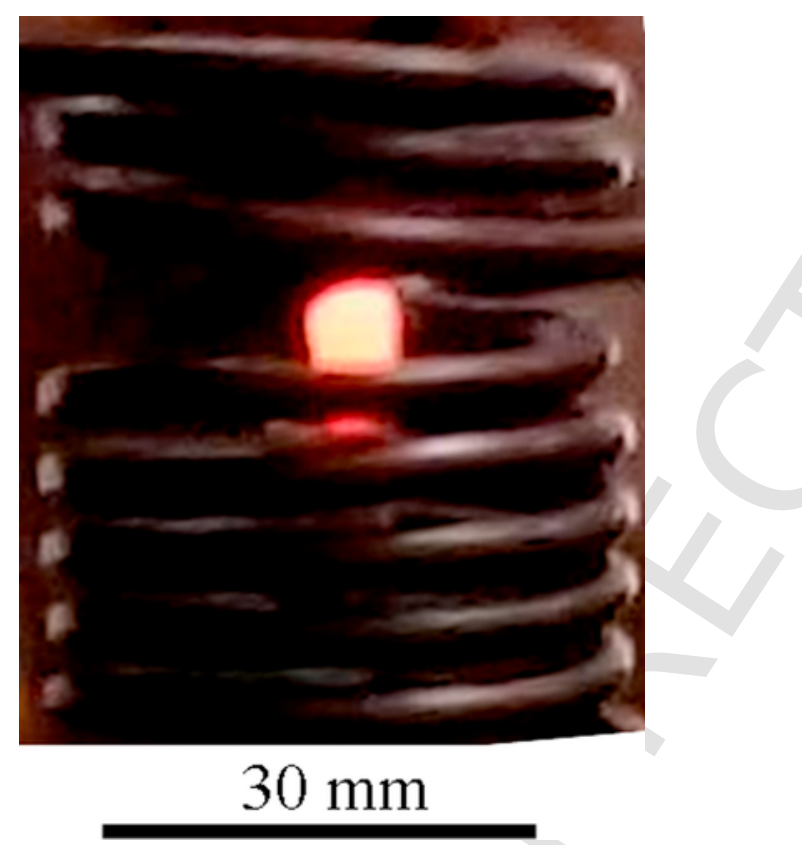

Fig. 1. Illustration of the coil used in the glass-fluxing experiment.

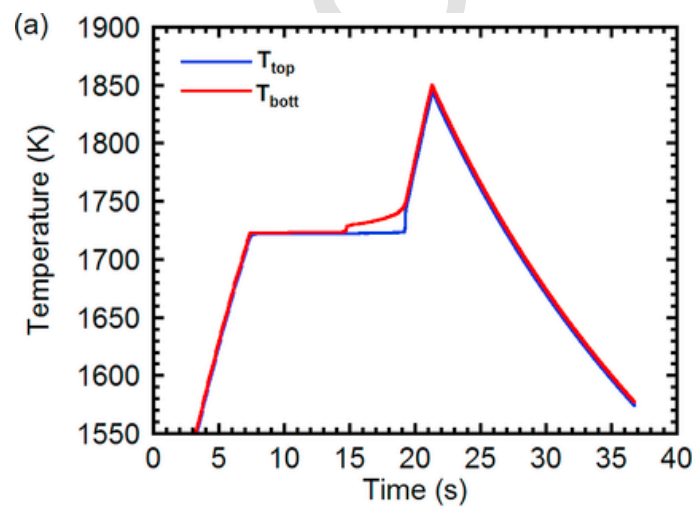

the recalescence event. Through the heating and cooling cycle, the temperature of the top surface of the sample was measured using a commercial single-color pyrometer at a wavelength of $0.9 \mu \mathrm{m}$ with an accuracy of $\pm 6 \mathrm{~K}$. A spectral emissivity of $\varepsilon=0.2$ was tentatively used in the measurement. After the experiment, the spectral emissivity of the sample was calibrated in such a way that an extrapolated recalescence temperature of $1692 \mathrm{~K}$ at a vanishing undercooling could match the equilibrium melting temperature of pure nickel, $1726 \mathrm{~K}$. This calibration gave a corrected spectral emissivity of $\varepsilon=0.185$. Meanwhile, the recalescence event on the upper surface of the sample was observed in-situ using a high-speed video camera to determine tip velocities in undercooled conditions. Such a melting and solidification cycle was repeated more than 30 times for studies of tip velocities over a wide spectrum of undercooling.

In the heating stage of the sample, the electrical current in the coil is not precisely known due to the complex circuit configuration of the radio frequency induction melting furnace. To determine this quantity, we assumed that a thermal balance was established between the sample and the surrounding media including the protective argon gas and glass melt. Then, we used a spectral collocation numerical method [11] to calculate induced currents, temperature distribution, and flow structure within the glass-fluxed sample. The induced currents were then correlated accurately with the electrical current in the coil. Simulations with coil currents ranging between $100 \mathrm{~A}$ and $200 \mathrm{~A}$ were conducted and the time dependent surface temperature was compared against experimental temperature curves (not shown here). Characteristic coil currents were obtained by calibrating against the time taken for isothermal melting and the time dependent thermal gradient, $d T / d t$, during heating and cooling. This comparison showed that the coil currents applied in individual melt-solidification cycles fall into the range between $130 \mathrm{~A}$ and $180 \mathrm{~A}$. The sample surface was determined to have a total hemispherical emissivity of $\varepsilon_{\text {total }}=0.24$, which is in excellent agreement with a measured value of 0.21 [14]. Fig. 2 illustrates the calculated surface temperatures of the sample during heating, melting, overheating and cooling using a typical coil current of $135 \mathrm{~A}$. The simulated cooling curve represents all undercoolings, i.e. recalescence can occur at any point after the temperature goes below the melting temperature. The surface temperature during recalescence is shown later on, in Section 3. Note that during heating and cooling, the top surface temperature always stays below the lower surface temperature. This difference is due to a stronger power absorption in the lower hemisphere of the sample during heating and due to additional radiation cooling from the top surface during cooling. The glass flux and sample holder were in direct thermal contact with the lower hemisphere of the sample. At this location there was heating from the coil due to the residual current in it, and the local temperature was higher

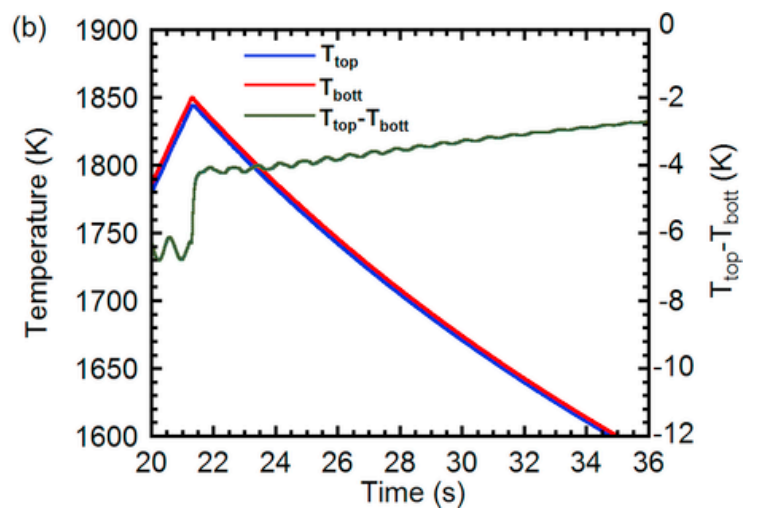

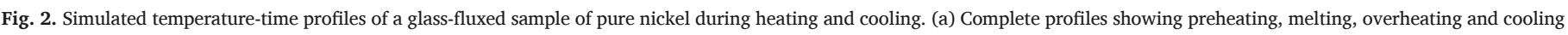
of the sample. (b) Zoomed-in profiles of the cooling stage showing the temperature difference between the top and bottom of the sample. 
than that of the upper hemisphere of the sample. As highlighted in Fig. $2 \mathrm{~b}$, a temperature difference of $\delta T \sim 7 \mathrm{~K}$ is reached at the end of the overheating stage. The difference declines to a value of $\delta T=4 \mathrm{~K}$ at the beginning of the cooling stage and still possesses a value of $\delta T \sim 3 \mathrm{~K}$ after a cooling time of $13 \mathrm{~s}$. Although the difference is comparable to the errors of $\pm 6 \mathrm{~K}$ of the measured temperatures, it can have a profound effect on the tip velocities at low undercoolings as discussed in Section 5.

Based on the simulated coil currents, we modeled the flow structure in the bulk volume of the glass-fluxed sample using an enthalpy-based method [15]. The results are shown in Fig. 3. When the sample is melted at a typical coil current of $135 \mathrm{~A}$, strong flow will form in the bulk volume of the sample. But the velocity field is highly non-uniform. The flow near the sample surface is strongest reaching a maximum velocity of $0.08 \mathrm{~m} / \mathrm{s}$. The flow in the sample center is distinctly weaker with a velocity of $\sim 0.05 \mathrm{~m} / \mathrm{s}$ only. When the coil current is reduced to $20 \mathrm{~A}$ for cooling, the maximum flow velocity will decline to a value of only $0.015 \mathrm{~m} / \mathrm{s}$. The flow structure is also different between the two cases. In the high current case, two toroidal vortices of somewhat similar sizes exist. One appears in the upper hemisphere and the other appears in the lower hemisphere. In the low current case, the flow structure is characterized by a single large vortex. This change is a consequence of the reduction in the Lorentz force, which is concentrated in the surface of the lower hemisphere of the sample.

\section{Modeling of dendritic structure and surface temperature}

We then modeled the temperature distribution and the dendritic structure during recalescence of the sample using the enthalpy-based method [14]. Fig. 4 illustrates snapshots of a recalescence event beginning with growth of an isothermal dendrite at an undercooling of $\Delta T=260 \mathrm{~K}$. The snapshots represent (111) growth relative to the observer. The simulated surface temperature shows a reasonable agreement with earlier experimental observations $[4,16]$. The surface of the sample is assumed to be adiabatic during this step because the recalescence process is very fast and radiation losses are negligible. At the end of the unconstrained growth process the curvature of the leading dendritic tip remains large and thus the surface temperature is still undercooled to satisfy local interfacial equilibrium conditions. The regions with a local positive curvature correspond to the undercooled tips and are incident to the surface of the sample. In contrast, the regions with a negative curvature correspond to the dendritic roots and lie below the surface of the sample. These roots, however, were not

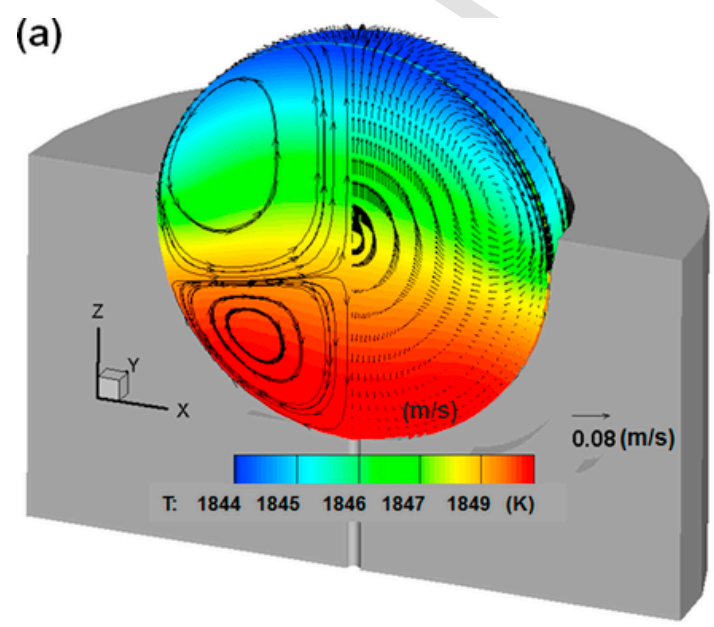

"seen" by the pyrometer during the glass-fluxing experiment. Due to such a spatial arrangement of the curvatures of the microstructure, the recalescence temperature measured by the pyrometer lies below the equilibrium melting temperature of the sample. This reduction in the recalescence temperature depends on undercooling as experimentally observed $[4,16]$. The larger the undercooling is, the smaller the dendritic spacing and thus the higher the tip curvature is.

The time scale of the solidification process will increase at the end of the recalescence event when the free energy of the sample is much lower. The internal thermal gradients of the sample are much lower than on the surface and thus, heat flux is significantly reduced when compared to the recalescence process. Consequently, the time scale of heat transport is larger when compared to the unconstrained growth during recalescence. In this case, the solidification process proceeds as a coarsening process which also has a much longer time scale than that of the unconstrained growth. The heat loss from radiation becomes significant again at this stage. Due to the large disparity in time scales, the current model used for the simulations requires development to simulate the entire process, which is planned in future work. Nevertheless, the current result highlights that calibrating temperature may lead to an underestimation of undercooling especially in the high undercooling regime.

\section{Correction of measured surface temperature}

The above numerical simulations reproduced the thermal history and revealed a large temperature difference in the bulk volume of the glass-fluxed sample. Although the simulations provided more details of the temperature difference, it is instructive to evaluate this quantity analytically. More critically, the analytical method allowed us to correct a systematic error of the measured surface temperature of the sample. The details of the analytical method and the correction of the measured temperature are given below.

Assuming the black body radiation loss balances the diffusion heat flux from the sample, one may write the following equation:

$q_{\text {diff }}=q_{\text {rad }}=K \frac{d T}{d z}=\varepsilon_{\text {total }} \sigma T^{4}$,

where $K$ is the thermal conductivity, $\varepsilon_{\text {total }}$ is the total hemispherical emissivity and $\sigma$ is the Stefan-Boltzmann constant. Using characteristic values of $K=54.7 \mathrm{~W} /(\mathrm{m} \cdot \mathrm{K})$ [17], $\varepsilon_{\text {total }}=0.21[14], \sigma=5.67 \times 10^{-8} \mathrm{~W} /$ $\left(\mathrm{m}^{2} \mathrm{~K}^{4}\right.$ ), and $T=1700 \mathrm{~K}$ gives $d T / d z=1.928 \times 10^{3} \mathrm{~K} / \mathrm{m}$, which corresponds to $\delta T=11.6 \mathrm{~K}$ over a distance of the sample size. At temperature of $T=1500 \mathrm{~K}$ this difference be-

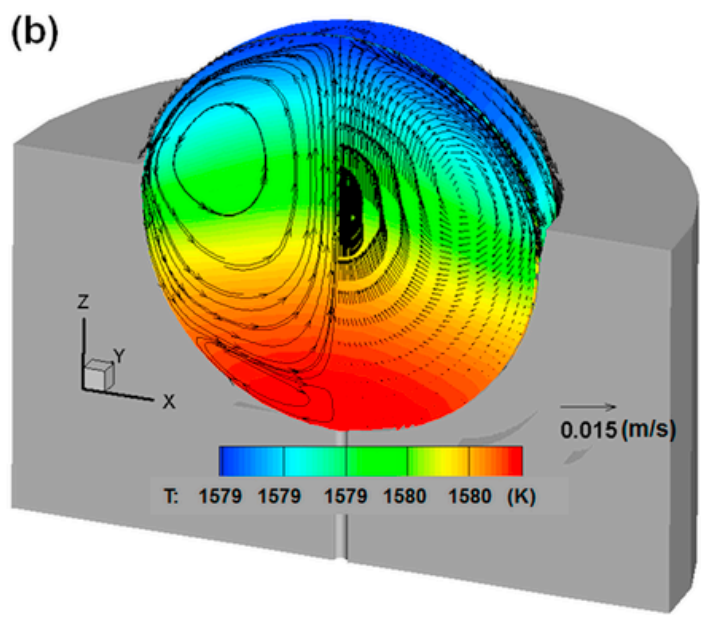

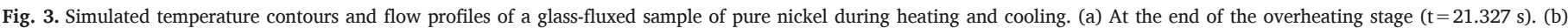
During the undercooling stage $(t=36.376 \mathrm{~s})$. 
(a)

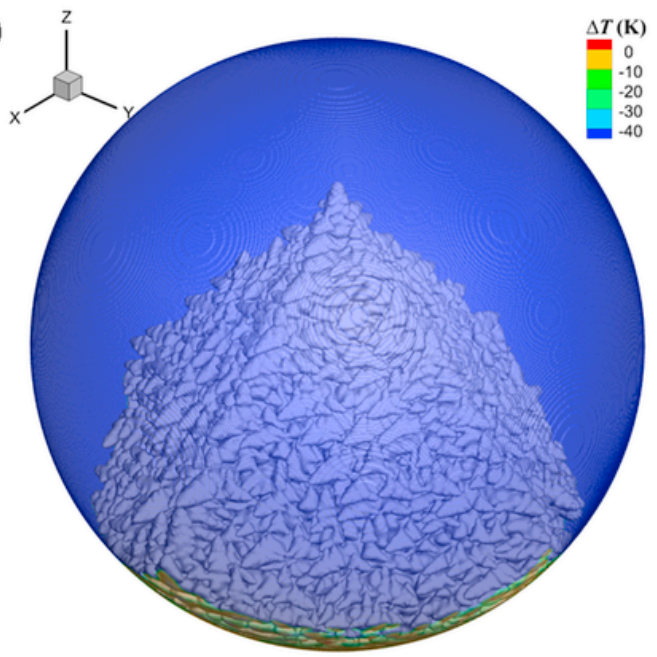

(b)

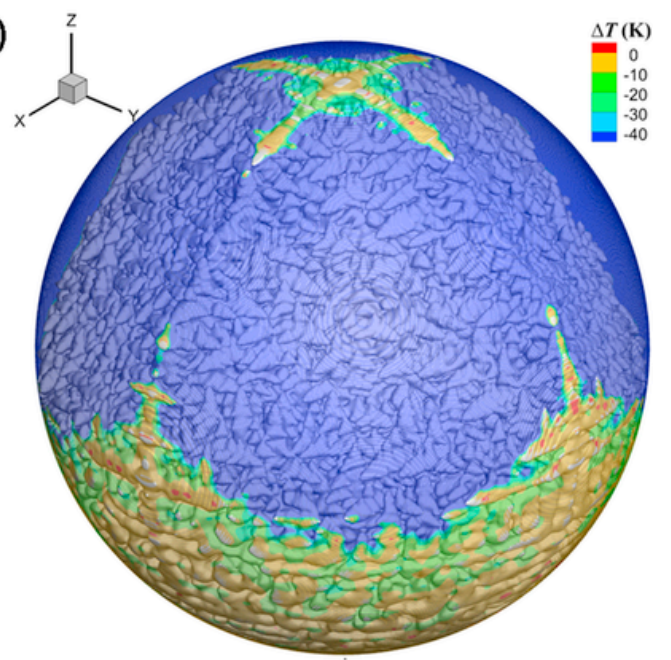

(c)

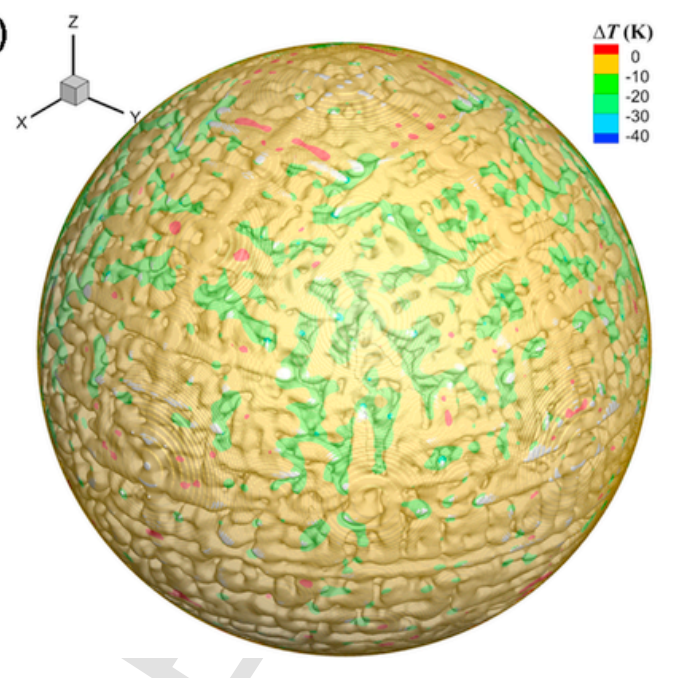

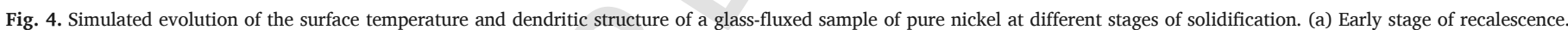
(b) Late stage of recalescence. (c) Coarsening process.

comes $\delta T=6.6 \mathrm{~K}$, agreeing well with the numerical simulations of Section 2. These results show that the difference in the surface temperature declines with rising undercooling.

Given that the tip velocity measurements can only be taken from the thermal front in the upper hemisphere of the glass-fluxed sample, the corresponding undercooling should be that of the upper hemisphere. Therefore, the influence of the lower hemisphere on the glass flux and sample holder will manifest as a net systemic error in the temperature measured by the pyrometer. The pyrometer will detect a higher temperature or a lower undercooling of the sample. To estimate this influence, we assumed that the relative temperature of the sample varies linearly in the $z$ direction from a high temperature $\delta T / 2$ to a low temperature of $-\delta T / 2$. In the experiment, the pyrometer was placed far away from the sample such that it would observe a projection of the hemispherical surface onto a circle given by

$T(r)=-\frac{\delta T}{2 r_{0}} \sqrt{r_{0}^{2}-r^{2}}$

where $r_{0}$ is the radius of the sample and $r$ is the radial component of the projected circle. The average value of this circle is the apparent temperature detected by the pyrometer from the sample and is given by

$\frac{1}{\pi r_{0}^{2}} \int_{0}^{r_{0}} \int_{0}^{2 \pi}-\frac{\delta T}{2 r_{0}} \sqrt{r_{0}^{2}-r^{2}} r d r d \theta=\frac{\delta T}{3}$.

This represents the ideal conditions where the sample holder has no effect on the pyrometer. Assuming that the sample holder can be represented by the average temperature of the lower hemisphere surface, the relative temperature of the holder becomes

$\frac{1}{2 \pi r_{0}^{2}} \int_{\pi / 2}^{\pi} \int_{0}^{2 \pi} \frac{\delta T}{2} r_{0} \cos \theta \sin \theta d \theta d \varphi=\frac{\delta T}{4}$,

where $\theta$ and $\varphi$ are the spherical angles of the sample. When the sample holder is included the increase in the observed temperature, $T_{i}$, from the ideal conditions by the pyrometer can be expressed as

$T_{i}=\delta T\left(\frac{A_{h} / 4-A_{s} / 3}{A_{s}+A_{h}}+\frac{1}{3}\right)$,

where $A_{s}$ and $A_{h}$ are the observed area of the sample and sample holder, respectively. Assuming a sample holder radius of $\sim 4.5 \mathrm{~mm}$, a sample radius of $\sim 3 \mathrm{~mm}$ and $\delta T=10 \mathrm{~K}$ gives an increase in the ob- 
served temperature $T_{i} \sim 5 \mathrm{~K}$. This influence will be most notable in the low undercooling region for two reasons. The first is that the $\delta T$ is the largest and a small increase in the measured apparent temperature gives a larger relative error when compared to the high undercooling region, and the second is that the residual mixing tends to reduce the thermal gradient with rising undercooling. For this influence, the measured undercoolings of the glass-fluxed sample should be corrected with an additional contribution due to the thermal gradient. It was determined that the additional contribution varies between $3 \mathrm{~K}$ and $5 \mathrm{~K}$ and decreases with rising undercooling. As shown below, this correction allowed us to conclude an improved agreement between theory and experiment.

\section{Modeling of dendritic tip velocities}

The above simulations revealed the flow structure and temperature distribution of the glass-fluxed sample before and during recalescence. Such knowledge allowed us to evaluate the fluid flow effects on the tip velocities quantitatively. In order to define the zero-flow condition independently, we referenced the measured tip velocities in electromagnetically levitated samples of high-purity nickel. The measurements by Funke et al. [8] showed that the tip velocities for undercoolings larger than $\Delta T=80 \mathrm{~K}$ are at least 10 times higher than the flow velocity of $0.3 \mathrm{~m} / \mathrm{s}$ in the electromagnetically levitated samples [11-13]. On the other hand, the tip velocities for undercoolings above $\Delta T=165 \mathrm{~K}$ show a deviation from a power law. Thus, the experimental data for undercoolings between $\Delta T=80 \mathrm{~K}$ and $\Delta T=165 \mathrm{~K}$ are supposed to be close to the tip velocities under ideal zero-flow conditions, i.e. pure diffusion conditions. Under this assumption, we fitted a recent theory on the three-dimensional dendritic growth [7] to the data. The theory predicts the tip selection parameter, $\sigma^{*}$, of an equiaxed dendrite growing from an undercooled dilute binary alloy melt with convection as

$\sigma^{*}=\frac{\sigma_{0} \beta^{7 / 4}}{1+b\left(\alpha \beta^{-3 / 4}\right)^{11 / 14}}\left[\frac{1}{\left(1+a_{1} \sqrt{\beta} P_{g}\right)^{2}}+\frac{1}{\left(1+a_{2} \sqrt{\beta} P_{g} D_{T} / D_{C}\right)^{2}}\right.$

where the selection constant $\sigma_{0}$ and the stability constant $b$ are material-dependent parameters, the stiffness $\beta=15 \varepsilon_{4}$ is a function of the surface tension anisotropy, $\varepsilon_{4}$, of the four-fold symmetry, $a_{1} \approx 0.381 \sigma_{0}, a_{2}$ $\approx 0.505 \sigma_{0}, D_{T}$ is the thermal diffusivity, $D_{C}$ is the chemical diffusivity, $m$ is the slope of liquidus, $k_{0}$ is the equilibrium partitioning coefficient, $Q$ is the latent heat, $C_{p}$ is the specific heat capacity. $P_{g}=V R /\left(2 D_{T}\right)$ is the growth Peclet number, where $R$ and $V$ are the dendritic tip radius and velocity, respectively. The parameter $\alpha$ is given by

$$
\alpha=\frac{d_{0} U a(\operatorname{Re})\left[1+D_{C} /\left(2 D_{T}\right)\right]}{4 R V\left[D_{C} /\left(2 D_{T}\right)+m C_{i}\left(1-k_{0}\right) c_{p} / Q\right]} .
$$

where $U$ is the incident flow velocity, $d_{0}$ is the thermocapillary length. The parameter $a(R e)$ is defined as

$a(\operatorname{Re})=\frac{\exp (-\operatorname{Re} / 2)}{E_{1}(\operatorname{Re} / 2)}$

where $E_{1}(q)=\int_{q}^{\infty} u^{-1} \exp (-u) d u$ is the first exponential integral function, $R e=R U_{\rho_{l}} / \mu$ is the Reynolds number, $\rho_{l}$ is the density of the liquid, and $\mu$ is the dynamical viscosity. $C_{i}$ is the concentration of solute atoms at the liquid/dendrite interface and can be expressed as
$C_{i}=\frac{C_{0}}{1-\left(1-k_{0}\right) \exp \left[\left(P_{f}+P_{g}\right) D_{T} / D_{C}\right] P_{g} I_{C}(\infty) D_{T} / D_{C}}$,

where $C_{0}$ is the bulk concentration of solute atoms, $P_{f}=U R /\left(2 D_{T}\right)$ is the flow Peclet number, and $I_{C}(\infty)$ is the concentration of solute atoms of the undercooled melt far from the liquid/dendrite interface. The $I_{C}(\infty)$ $=\left.I_{C}(\eta)\right|_{\eta \rightarrow \infty}$ is given by

$I_{\mathrm{C}}(\eta)=\int_{1}^{\eta} \exp \left[2 P_{f} \frac{D_{T}}{D_{C}} \int_{1}^{\eta^{\prime}} \frac{g\left(\eta^{\prime \prime}\right)}{\sqrt{\eta^{\prime \prime}}} d \eta^{\prime \prime}-\left(P_{f}+P_{g}\right) \frac{D_{T}}{D_{C}} \eta^{\prime}\right] \frac{d \eta^{\prime}}{\eta^{\prime}}$,

where the hydrodynamic function $g\left(\eta^{\prime \prime}\right)$ is given by

$g\left(\eta^{\prime \prime}\right)=\frac{\sqrt{\eta^{\prime \prime}} E_{1}\left(\operatorname{Re} \eta^{\prime \prime} / 2\right)}{2 E_{1}(\operatorname{Re} / 2)}+\frac{\exp (-\operatorname{Re} / 2)-\exp \left(-\operatorname{Re} \eta^{\prime \prime} / 2\right)}{\sqrt{\eta^{\prime \prime}} \operatorname{Re} E_{1}(\operatorname{Re} / 2)}$.

The undercooling includes four contributions:

$\Delta T=\Delta T_{T}+\Delta T_{C}+\Delta T_{R}+\Delta T_{K}$,

where $\Delta T_{T}=P_{g} \exp \left(P_{g}+P_{f}\right) I_{T}(\infty) Q / C_{p}$ is the thermal undercooling, $\Delta T_{R}=2 d_{0} Q /\left(C_{p} \rho_{1}\right), \Delta T_{k}=V / \mu_{k}$ is the thermal undercooling, where $\mu_{k}$ is the interfacial kinetic coefficient. The constitutional undercooling $\Delta T_{C}$ is given by

$\Delta T_{C}=\frac{m C_{0}\left(1-k_{0}\right) P_{C g} \exp \left(P_{C g}+P_{C f}\right) I_{C}(\infty)}{1-\left(1-k_{0}\right) P_{C g} \exp \left(P_{C g}+P_{C f}\right) I_{C}(\infty)}$,

where $P_{C g}=V R /\left(2 D_{C}\right)$ and $\mathrm{P}_{C f}=U R /\left(2 D_{C}\right)$ are chemical Peclet numbers. The function $I_{T}(\infty)=\left.I_{T}(\eta)\right|_{\eta \rightarrow \infty}$ is given by

$I_{\mathrm{T}}(\eta)=\int_{1}^{\eta} \exp \left[2 P_{f} \int_{1}^{\eta^{\prime}} \frac{g\left(\eta^{\prime \prime}\right)}{\sqrt{\eta^{\prime \prime}}} d \eta^{\prime \prime}-\left(P_{f}+P_{g}\right) \eta^{\prime}\right] \frac{d \eta^{\prime}}{\eta^{\prime}}$,

Using Eqs. (6)-(14) we calculated the tip velocities in undercooled melts of pure nickel without any impurities first. In order to determine the unknown parameters $\sigma_{0}$, we fitted the theory to the measured tip velocities in the electromagnetically levitated samples [8] using a least square logarithm and the parameters listed in Table 1.

Table 1

Materials parameters used in the calculations of dendritic tip velocities.

\begin{tabular}{lll}
\hline Parameter & Value & References \\
\hline Thermal diffusivity, $D_{T}\left(\mathrm{~m}^{2} / \mathrm{s}\right)$ & $1.07 \times 10^{-5}$ & {$[17]$} \\
Hypercooling limit, $Q / C_{p}(\mathrm{~K})$ & 418 & {$[10]$} \\
Thermocapillary length, $d_{0}(\mathrm{~m})$ & $4.92 \times 10^{-10}$ & {$[10]$} \\
Liquid density, $\rho_{l}\left(\mathrm{~kg} \cdot \mathrm{m}^{-3}\right)$ & 7900 & {$[10]$} \\
Dynamic viscosity, $\mu(\mathrm{Pa})$ & $5.64 \times 10^{-3}$ & {$[10]$} \\
Surface tension anisotropy, $\varepsilon_{4}(-)$ & 0.018 & {$[10]$} \\
Kinetic coefficient, $\mu_{k}\left(\mathrm{~m} \cdot \mathrm{s}^{-1} \cdot \mathrm{K}^{-1}\right)$ & 0.66 & {$[18]$} \\
Bulk concentration, $C_{0}(\mathrm{at} \%)$ & $1 \times 10^{-3}$ & Present work \\
Chemical diffusivity, $D_{C}\left(\mathrm{~m}^{2} / \mathrm{s}\right)$ & $5.0 \times 10^{-9}$ & Present work \\
Partitioning coefficient, $k_{0}(\mathrm{at} . / \mathrm{at})$. & $1.5 \times 10^{-2}$ & Present work \\
Slope of liquidus, $m(\mathrm{~K} / \mathrm{at} \%)$ & $1 \times 10^{-3}$ & Present work \\
Selection constant, $\sigma_{0}(-)$ & 0.191 & Present work \\
Stability constant, $b(-)$ & 0.1 & {$[7,10]$}
\end{tabular}


In this logarithm, we defined a percentage deviation of the measured tip velocities with respect to the calculated tip velocities for each undercooling. Unlike the previous calculations [10], the present calculation adopted a corrected interfacial kinetic coefficient of $\mu_{k}$ $=0.66 \mathrm{~ms}^{-1} \mathrm{~K}^{-1}[18]$. Then, the unknown parameter $\sigma_{0}$ was determined to have a value of 0.191 , which is nearly twice larger than the previously determined value [10]. The root of the mean square of the percentage deviation in the undercooling range $80-165 \mathrm{~K}$ is as low as $9.1 \%$. When compared with the previous fitting [10], this new fitting produces an extensive agreement between the theory and the measured tip velocities up to a maximum undercooling of $\Delta T=259 \mathrm{~K}$. However, as shown in Fig. 5, the calculated tip velocities for undercoolings below $\Delta T=80 \mathrm{~K}$ under the zero-flow conditions become larger than those measured in the electromagnetically levitated samples [8]. This difference can be ascribed to a flow of $0.3 \mathrm{~m} / \mathrm{s}$ in the same direction of growth, which slows down heat transport ahead of the growing tips and thus reduces the tip velocities. Although the flow direction is opposite to the generally accepted, the flow velocity is yet consistent with the prediction of magnetohydrodynamics simulations

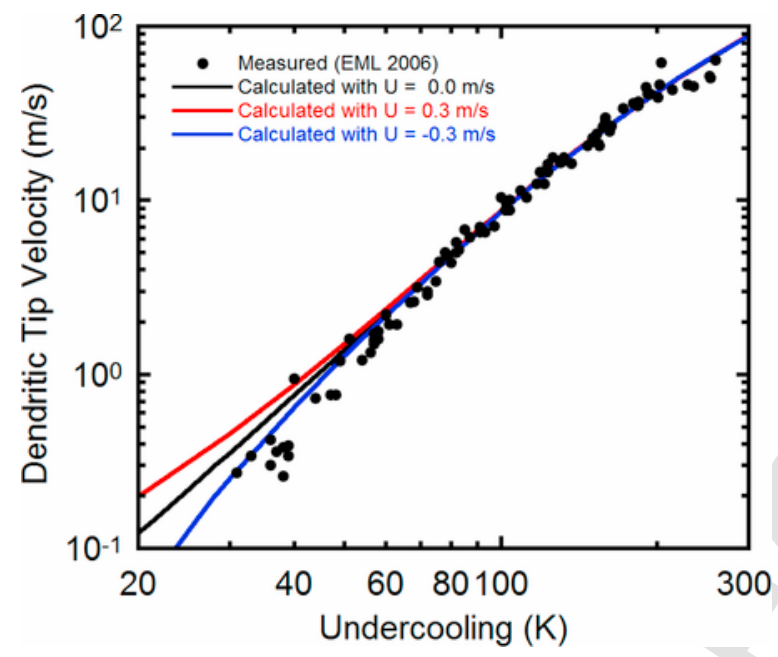

Fig. 5. Calculated dendritic tip velocities in electromagnetically levitated samples of pure nickel under different flow conditions. The positive and negative signs of the flow velocities correspond to flows in the incident and same directions of dendritic growth, respectively. The experimental data are taken from Ref. 8 and imposed for comparison.

(a)

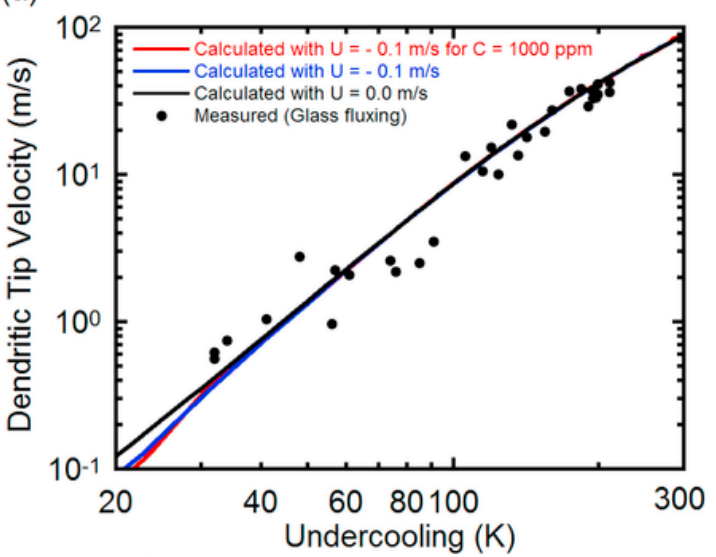

[11-13]. Such additional agreement supports the reliability of the present calculation.

We then calculated the tip velocities in the glass-fluxed nickel sample using the same theory [7]. We considered a flow of $0.1 \mathrm{~m} / \mathrm{s}$ in the same direction of growth. This flow velocity is by $20 \%$ larger than the predicted flow velocity of $0.08 \mathrm{~m} / \mathrm{s}$ in the molten sample (see Section 2 ), and thus can be referred to as an upper limit of the real flow velocity. To explore the suggested effect of impurities $[8,10]$, we also calculated tip velocities in the presence of a strong partitioning impurity at a high concentration level of $C_{0}=1000 \mathrm{ppm}$. As shown in Fig. 6a, the calculated tip velocities for undercoolings below $\Delta T=50 \mathrm{~K}$ are clearly below the measured tip velocities irrespective of any impurity consideration. Therefore, it can be concluded that neither the flow effect nor the impurity effect are the main reasons for the large discrepancy between the glass-fluxing experiments and the theory. Rather, it can be attributed to the errors in the measured temperature of the glass-fluxed sample as explained in Section 4. If the errors are corrected, the discrepancy can be eliminated. As shown in Fig. $6 \mathrm{~b}$, the previously measured tip velocities of the glass-fluxed sample are replotted as a function of the corrected undercooling. The experimental data shows a fair agreement with the predictions of the theory even if the contributions of the flow and impurity effects are neglected. In this sense, the glass-fluxing experiments enable measurements of tip velocities in a microgravity-mimicking environment, provided that melt undercooling of the sample is determined with a high reliability.

\section{Conclusions}

The melt flow, thermal history and dendritic structure of the glass-fluxed nickel sample have been simulated numerically. The simulations have reproduced the experimentally observed surface temperature of the sample through heating and cooling. The simulations have revealed a maximum flow velocity of $0.08 \mathrm{~m} / \mathrm{s}$ and $0.015 \mathrm{~m} / \mathrm{s}$ in the overheated sample and in the undercooled sample, respectively. Such flow velocities are too small to produce any significant effects on the tip velocities which are measurable using a high-speed camera. However, the simulations have revealed a large thermal gradient crossing the sample size, which can cause an underestimation of the real undercooling for dendritic growth during recalescence. By correcting this underestimation, we have concluded an improved agreement between the theory of three-dimensional dendritic growth and the glass-fluxing experiments.

(b)

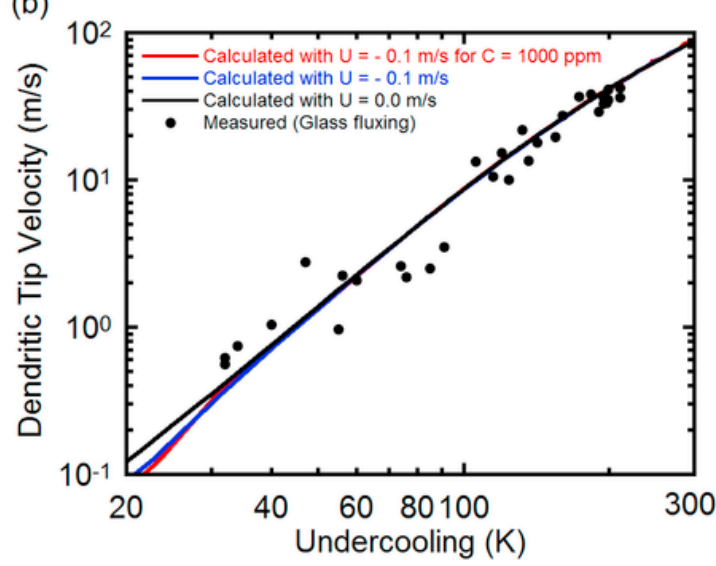

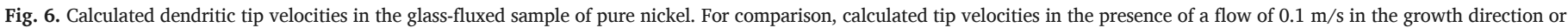

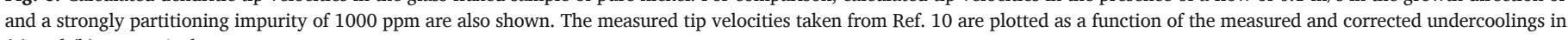
(a) and (b), respectively. 


\section{Acknowledgement}

The authors thank S. Cai and Z. Zhai for editing computer codes for calculations of dendritic tip velocities. J. Gao acknowledges support by the National Natural Science Foundation of China (51071043 and 51211130113), Fundamental Research Funds for Central Universities (N130509001), and the Overseas Expert Program of the International Office of Northeastern University (X2015001). A. Kao and K. Pericleous acknowledge support by the International Exchanges Scheme of the Royal Society of the United Kingdom. D. V. Alexandrov acknowledges support from the Russian Science Foundation (Project No. 16-11-10095). P. K. Galenko acknowledges support by German Research Foundation (DFG Project RE 1261/8-2) and Russian Foundation for Basic Research (RFBR Project No. 14-29-10282).

\section{References}

[1] J.S. Langer, Instabilities and pattern formation in crystal growth, Rev. Mod. Phys. 52 (1980) 1-28.

[2] R. Trivedi, W. Kurz, Dendritic growth Inter. Mater. Rev. 39 (1994) 49-74.

[3] J.J. Hoyt, M. Asta, A. Karma, Atomistic and continuum modeling of dendritic solidification, Mater. Sci. Eng. R 41 (2003) 121-163.

[4] J. Gao, Z.N. Zhang, Y.K. Zhang, C. Yang, Measurements of crystal growth dynamics in glass-fluxed melts, in: D.M. Herlach, D.M. Matson (Eds.), Solidification of Undercooled Melts, Wiely VCH, Weinheim, 2012, pp. 281-303.

[5] H. Müller-Krumbhaar, J.S. Langer, Theory of dendritic growth-III. Effects of surface tension, Acta Metall. 26 (1978) 1697-1708.

[6] E.A. Brener, Effects of surface energy and kinetics on the growth of needle-like dendrites, J. Cryst. Growth 99 (1990) 165-170.
[7] D.V. Alexandrov, P.K. Galenko, Dendrite growth under forced convection: analysis methods and experimental tests, Phys.-Uspekhi 57 (2014) 771-786.

[8] O. Funke, G. Phanikumar, P.K. Galenko, L. Chernova, S. Reutzel, M. Kolbe, D.M. Herlach, Dendrite growth velocity in levitated undercooled nickel melts, J. Cryst. Growth 297 (2006) 211-222.

[9] S.-C. Huang, M.E. Glicksman, Overview 12: fundamentals of dendritic solidification - I. steady-state tip growth, Acta Metall. 29 (1985) 701-715.

[10] J. Gao, M.K. Han, A. Kao, K. Pericleous, D.V. Alexandrov, P.K. Galenko, Dendritic growth velocities in an undercooled melt of pure nickel under static magnetic fields: a test of theory with convection, Acta Mater. 103 (2016) 184-191.

[11] V. Bojarevics, K. Pericleous, Modeling electromagnetically levitated liquid droplet oscillations ISIJ Inter 43 (2003) 890-898.

[12] R.W. Hyers, Fluid flow effects in levitated droplets, Meas. Sci. Technol. 16 (2005) 391-401.

[13] J.H. Lee, D.M. Matson, S. Binder, M. Kolbe, D. Herlach, R.W. Hyers, Magnetohydrodynamic modeling and experimental validation of convection inside electromagnetically levitated Co-Cu droplets, Metall. Mater. Trans. B 45 (2014) 1018-1023.

[14] A.J. Rulison, W.-K. Rhim, A noncontact measurement technique for the specific heat and total hemispherical emissivity of undercooled refractory materials, Rev. Sci. Instrum. 65 (1994) 695-700.

[15] A. Kao, K. Pericleous, A numerical model coupling thermoelectricity, magnetohydrodynamics and dendritic growth, J. Alog. Comput. Technol. 6 (2012) 173-201.

[16] J. Gao, Z. N. Zhang, Y. J. Zhang, Measurements of dendritic growth velocities in undercooled melts of pure nickel under static magnetic fields, R. Hyers, V. Bojarevis, J. Downey, H. Henein, D. Matson, A. Seidel, D. Voss, M. San Soucie (Eds.), Proceedings of a Symposium on Materials Research in Microgravity 2012 Held at the 141st TMS Annual Meeting and Exhibition, Orlando (2012) pp. 72-79.

[17] T. Nishi, H. Shibata, H. Ohta, Y. Waseda, Thermal conductivities of molten iron, cobalt, and nickel by laser flash method, Metall. Mater. Trans. A 34 (2003) 2801-2807.

[18] J. Monk, Y. Yang, M.I. Mendelev, M. Asta, J.J. Hoyt, D.Y. Sun, Determination of the crystal-melt interface kinetic coefficient from molecular dynamics simulations, Model. Simul. Mater. Sci. Eng. 18 (2010) 015004. 